\title{
Theoretical and Experimental Determination of the Sound-Absorbing Property Class of Acoustic Barriers
}

\author{
J. TURKIEWICZ* AND G. WSZOEEK
}

AGH University of Science and Technology, Faculty of Mechanical Engineering and Robotics, Department of Mechanics and Vibroacoustics, Al. A. Mickiewicza 30, 30-059 Krakow, Poland

\begin{abstract}
The currently applicable PN-EN 1793-1 standard concerning anti-noise devices on roads recommends for acoustic barriers built along traffic routes to have panels characterized by good sound-absorbing properties. The sound-absorbing properties of these panels (wall elements) are to be specified by giving the values of the singlenumber sound absorption evaluation index $D L_{\alpha}$ and the absorption property class determined in accordance with the PN-EN 1793-3:2001 standard. Continuous regular acoustic studies of new materials heretofore not used in anti-noise protections are conducted in the Department of Mechanics and Vibroacoustics at AGH. This paper presents the findings of research that started with different porous materials physical absorption coefficient tests. The purpose of these preliminary tests was to pick out materials with best sound-absorbing properties from the group of acoustically tested materials. The chosen materials could be used as a sound-absorbing lining on panels (wall elements) used in the design of acoustic barriers. On the basis of the obtained results, two materials with values of the average physical sound absorption coefficient higher than $0.5\left(\alpha_{\text {favg }}>0.5\right)$ were picked out for further acoustic tests. This paper presents results of studies that had the final effect consisting in determining the values of the single-number sound absorption evaluation index $D L_{\alpha}$ and the absorption property class for two prototype panels of an acoustic barrier the sound-absorbing lining of which was made up of materials chosen after preliminary acoustic studies. The determination of sound-absorbing property classes was carried out both theoretically and experimentally. In order to be possible to reduce the costs of acoustic tests in the future, particularly of new materials in regard to which there is a margin of uncertainty of whether their sound-absorbing properties meet expectations, the authors determined sound-absorbing property classes of acoustic barriers theoretically and then compared theoretical results with the results of experimental tests.
\end{abstract}

DOI: 10.12693/APhysPolA.125.A-127

PACS: $43.55 . \mathrm{Ev}, 43.50 . \mathrm{Gf}$

\section{Introduction}

Over the last several years, many various types of acoustic barriers have been designed and constructed. The primary task of these barriers is to produce an acoustic shadow, that is, an area through which sound waves fail to propagate directly from a sound source. Currently, the most often constructed acoustic barriers have the purpose of acoustic protection of the external environment from one of the most onerous noise sources, i.e. traffic routes. From the perspective of effective protection against noise in urban housing estates, particularly those with continuous two-sided development, the constructed acoustic barriers should have good sound-absorbing properties. Such barriers, called the sound-absorbing barriers, can absorb sound waves from one side (the noise source side) or from two sides (both the noise source and the protected buildings' side) and they are characterized by a good sound absorption coefficient. Such barriers reduce the reverberation of the area around a roadway which increases barrier efficiency at the same time.

The currently applicable PN-EN 1793-1 standard [1] recommends for acoustic barriers constructed along traffic routes to have an implemented sound-absorbing element for the purpose of reducing the burden resulting

\footnotetext{
*corresponding author; e-mail: turkiewi@imir.agh.edu.pl
}

from the reflection of sound from this barrier. With this in mind, the standard recommends the determination of the sound-absorbing property class of the applied acoustic barrier [2]. The acoustic property class depends on the value of the single-number sound absorption evaluation index $D L_{\alpha}$ expressed in decibels. The value of this index is calculated as follows:

$$
D L_{\alpha}=-10 \log _{10}\left|1-\frac{\sum_{i=1}^{18} \alpha_{s i} 10^{0.1 L_{i}}}{\sum_{i=1}^{18} 10^{0.1 L_{i}}}\right|
$$

where $\alpha_{s i}$ is the sound absorption coefficient in the $i$ th $1 / 3$ octave frequency band and $L_{i}$ is the normalized sound level $\mathrm{A}$ (in $\mathrm{dB}$ ) of roadway noise in the $i$-th $1 / 3$ octave frequency band defined according to the PN-EN 1793-3 standards [2].

Based on the values of the $D L_{\alpha}$ index, sound-absorbing devices are classified in terms of their absorption properties in accordance with the PN-EN 1793-3 standard [2]. The classes and the index values corresponding to them are presented in Table I.

Absorption property classes [2]. TABLE I

\begin{tabular}{c|c}
\hline \hline Class & $D L_{\alpha},[\mathrm{dB}]$ \\
\hline $\mathrm{A} 0$ & Not determined \\
$\mathrm{A} 1$ & $<4$ \\
$\mathrm{~A} 2$ & 4 to 7 \\
$\mathrm{~A} 3$ & 8 to 11 \\
$\mathrm{~A} 4$ & $>11$
\end{tabular}


Determination of the absorption class is helpful when choosing the type of an acoustic barrier according to its specific location in the field.

The value of absorption coefficient $\alpha_{s i}$ present in the above formula is the value of the coefficient determined using the reverberation method. In order to determine the value of the reverberant sound absorption coefficient of a material, a sample of the material with surface area from 10 to $12 \mathrm{~m}^{2}$ must be used. Acoustic tests of this type are expensive, and in the case of new materials, the obtained result may prove to be insufficient. With this fact in mind, the authors used the value of the physical absorption coefficient of the studied materials (for determination of which a material sample with a diameter of $10 \mathrm{~cm}$ and $3 \mathrm{~cm}$ is sufficient), and based on these results, the authors theoretically determined the absorption property classes of these materials $[3,4]$. Using the physical absorption coefficient of the studied materials, the value of the reverberant sound absorption coefficient was determined with the application of a chart according to Morse' as shown in [5]. Next, using the reverberant sound absorption coefficient determined in this way, the $D L_{\alpha}$ index was calculated and the absorption property class of the panel with the studied material used as the sound-absorbing lining was determined. Because results of these acoustic tests and calculations conformed to expectations as to the obtained absorption class, the results were verified through measurements. Samples with appropriately large surfaces were made from the studied materials, acoustic tests were performed on prototype panels in a reverberation chamber, and the absorption property classes for these panels were obtained experimentally in accordance with the methodology provided for this type of testing $[1,2,6]$. Results of acoustic tests obtained in this manner have provided answers to two questions: can the studied panels be applied in the design of acoustic barriers (do they meet requirements of the standard) and can the absorption property class of an acoustic barrier be determined using a partially theoretical approach.

\section{The arrangement of experiments}

Acoustic tests performed at the Department of Mechanics and Vibroacoustics at AGH pertained to many prototype acoustic panels (wall elements). In many cases, very good compliance of results was obtained, except for slight differences in the value of $D L_{\alpha}$ which had no influence on the determined absorption class. In this paper, the authors present indicative results for only two types of acoustic panels: one made of expanded clay aggregate and the other made of chip concrete. Results of tests carried out for other materials will be published elsewhere.

The first of the tested acoustic barriers was made from expanded clay aggregate (Fig. 1). Expanded clay aggregate consists of light porous granules obtained through firing of swelling loams and clayey clays in rotary furnaces at about $1200{ }^{\circ} \mathrm{C}$, is extremely resistant to chemical and atmospheric factors and fungi, non-flammable and frost resistant, shows low absorbability, and is classified as one of the most ecological materials (Fig. 1a). Earlier preliminary acoustic tests of this expanded clay aggregate proved that it can be classified among the narrow-band sound-absorbing materials [3]. It can be useful as an additional sound-absorbing layer in barriers, e.g. in the form of barrier wall elements.

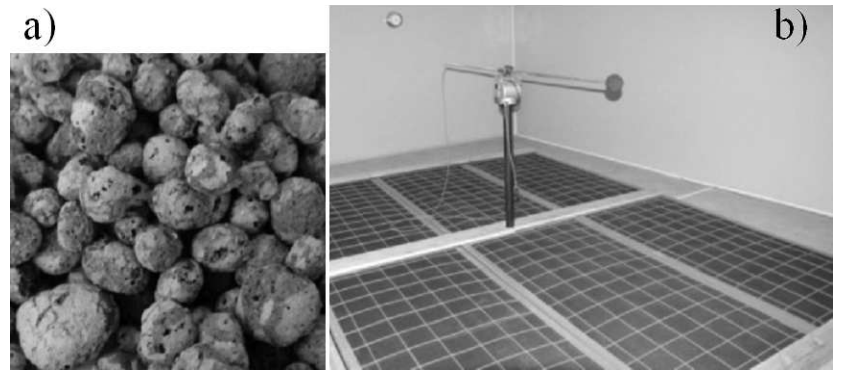

Fig. 1. Expanded clay aggregate: (a) photograph of expanded clay aggregate - grain fraction: $4-10 \mathrm{~mm}$, grain shape: irregular oval; (b) view of a barrier sample with panels filled with expanded clay aggregate - tests in the reverberation chamber.

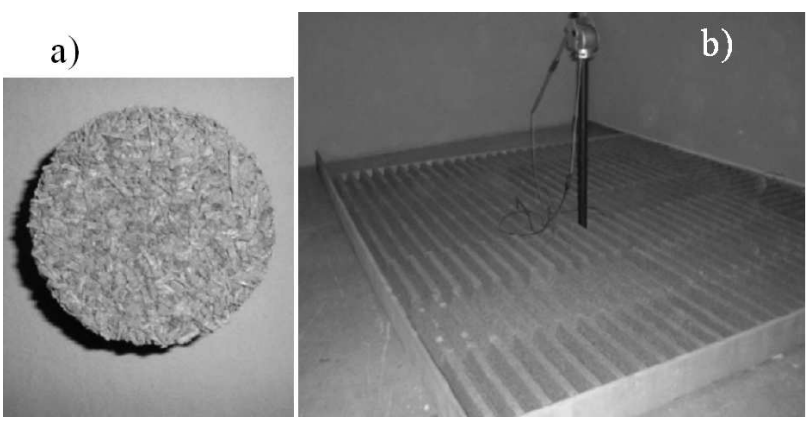

Fig. 2. Chip concrete: (a) photograph of a chip concrete sample tested in the Kundt tube; (b) view of a barrier sample with chip concrete panels - tests in the reverberation chamber.

The second tested panel was made from a material called the chip concrete (Fig. 2). Samples of the material that were used for acoustic tests were made of a type of material called composite. The concept of a composite material is used to describe a material that is made up of at least two different components combined at the macroscopic level. Most composite materials are made up of two phases, of which a continuous phase called the matrix surrounds the second phase, the so-called dispersed phase. The resultant properties of a composite are dependent on properties of the component phases, their fractions in the total volume of the composite, the method of distribution of the dispersed phase in the matrix, as well as on the geometrical properties of the dispersed phase.

Acoustic tests of composite materials are conducted throughout the world. For example, the absorption properties of samples made from jute fiber and of composites made on the base of these fibers with latex of different volumetric masses and sample thicknesses have been tested [7]. 
Preliminary pilot acoustic tests of the composite material called the chip concrete were also conducted at the Department of Mechanics and Vibroacoustics at AGH. Results of these tests show that this material possesses sound-absorbing properties -the average value of the physical sound absorption coefficient $\alpha_{p h, a v g}>0.5$.

\section{Test results}

Results of the conducted acoustic tests are presented in chart and tabular form. Table II presents results for expanded clay aggregate, and Table III presents results for chip concrete. The results in the tables include: results from prior tests of the physical sound absorption coefficient, results of reverberant absorption coefficient values for the same materials obtained on the basis of conversion of the physical absorption coefficient in accordance with the tabular chart found in [5], and results of the reverberant sound coefficient for barrier samples made from these materials that were tested in the reverberation chamber. The last row of each table contains $D L_{\alpha}$ indices as well as the absorption property classes for values of the reverberation coefficient converted from the physical coefficient and obtained from tests in the reverberation chamber.

\section{TABLE II}

Results of tests and calculations of sound absorption coefficients for expanded clay aggregate.

\begin{tabular}{c|c|c|c}
\hline \hline \multirow{2}{*}{$\begin{array}{c}\text { Frequency, } \\
\mathrm{Hz}\end{array}$} & \multicolumn{3}{|c}{$\begin{array}{c}\text { Sound absorption coefficient } \\
\text { of expanded clay aggregate }\end{array}$} \\
\cline { 2 - 4 } & Physical & \multicolumn{2}{|c}{ Reverberant from } \\
\cline { 2 - 3 } & & physical & tests \\
\hline 100 & 0.05 & 0.20 & 0.32 \\
125 & 0.08 & 0.20 & 0.27 \\
160 & 0.06 & 0.20 & 0.30 \\
200 & 0.06 & 0.20 & 0.26 \\
250 & 0.04 & 0.20 & 0.24 \\
315 & 0.10 & 0.20 & 0.23 \\
400 & 0.13 & 0.35 & 0.29 \\
500 & 0.19 & 0.85 & 0.40 \\
630 & 0.31 & 1.00 & 0.49 \\
800 & 0.54 & 0.93 & 0.63 \\
$1 \mathrm{k}$ & 0.87 & 0.65 & 0.74 \\
$1.25 \mathrm{k}$ & 0.83 & 0.35 & 0.77 \\
$1.6 \mathrm{k}$ & 0.59 & 0.75 & 0.66 \\
$2 \mathrm{k}$ & 0.40 & 0.35 & 0.54 \\
$2.5 \mathrm{k}$ & 0.47 & 0.93 & 0.53 \\
$3.15 \mathrm{k}$ & 0.67 & 0.75 & 0.50 \\
$4 \mathrm{k}$ & 0.95 & 1.00 & 0.41 \\
$5 \mathrm{k}$ & 0.56 & 0.89 & 0.34 \\
$\alpha_{a v}$ & 0.51 & 0.54 & 0.44 \\
$D L_{\alpha},[\mathrm{dB}]$ & & $4.34=4$ & $3.53=4$ \\
$\mathrm{Class}$ & & $\mathrm{A} 2$ & $\mathrm{~A} 2$ \\
& & &
\end{tabular}

The charts presented in Figs. 3 and 4 illustrate the curves of the reverberant sound absorption coefficient in 1/3-octave frequency bands for the tested expanded clay aggregate and chip concrete, respectively.

\section{TABLE III}

Results of tests and calculations of sound absorption coefficients for chip concrete.

\begin{tabular}{c|c|c|c}
\hline \multirow{2}{*}{$\begin{array}{c}\text { Frequency, } \\
\mathrm{Hz}\end{array}$} & \multicolumn{3}{|c}{$\begin{array}{c}\text { Sound absorption coefficient } \\
\text { of chip concrete }\end{array}$} \\
\cline { 2 - 4 } & Physical & \multicolumn{2}{|c}{ Reverberant from } \\
\cline { 2 - 4 } & & physical & tests \\
\hline 100 & 0.23 & 0.42 & 0.17 \\
125 & 0.22 & 0.41 & 0.29 \\
160 & 0.23 & 0.43 & 0.48 \\
200 & 0.28 & 0.48 & 0.59 \\
250 & 0.32 & 0.55 & 0.62 \\
315 & 0.45 & 0.70 & 0.63 \\
400 & 0.64 & 0.87 & 0.73 \\
500 & 0.89 & 1.00 & 0.85 \\
630 & 0.97 & 1.00 & 0.99 \\
800 & 0.87 & 1.00 & 1.00 \\
$1 \mathrm{k}$ & 0.71 & 0.92 & 0.93 \\
$1.25 \mathrm{k}$ & 0.51 & 0.75 & 0.88 \\
$1.6 \mathrm{k}$ & 0.73 & 0.93 & 0.76 \\
$2 \mathrm{k}$ & 0.74 & 0.94 & 0.77 \\
$2.5 \mathrm{k}$ & 0.30 & 0.50 & 0.78 \\
$3.15 \mathrm{k}$ & 0.64 & 0.86 & 0.82 \\
$4 \mathrm{k}$ & 0.50 & 0.75 & 0.83 \\
$5 \mathrm{k}$ & 0.46 & 0.71 & 0.80 \\
$\alpha_{a v}$ & 0.54 & 0.73 & 0.72 \\
$D L_{\alpha},[\mathrm{dB}]$ & & $8.27=8$ & $7.72=8$ \\
Class & & $\mathrm{A} 3$ & $\mathrm{~A} 3$ \\
& & &
\end{tabular}

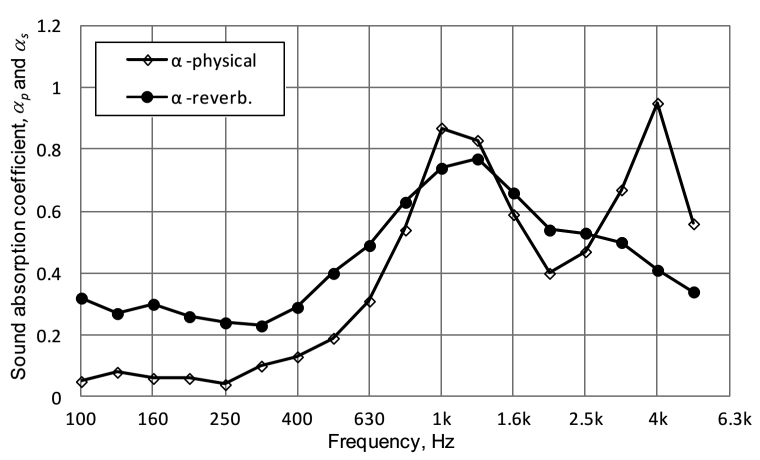

Fig. 3. Sound absorption curve for expanded clay aggregate granulate, obtained from results of physical and reverberant sound coefficient tests.

Slight differences in obtained $D L_{\alpha}$ values can be observed based on results of the conducted tests presented in the tables. However, this does not have a significant influence on the determination of sound absorption property classes because according to the guidelines of the standard, after rounding, the results indicate the same absorption property class in both cases.

\section{Concluding remarks}

The panels applied in acoustic barriers, which are most often constructed along traffic routes, must have a specific absorption property class in accordance with the ap- 


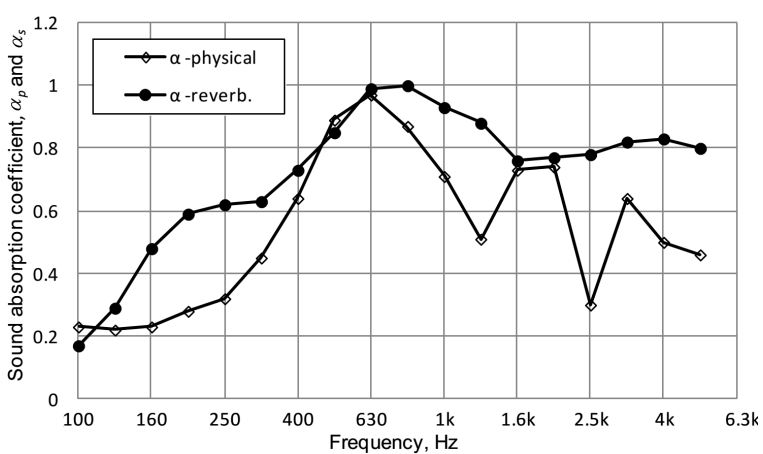

Fig. 4. Sound absorption curve for chip concrete, obtained from results of physical and reverberant sound coefficient tests.

plicable PN-EN 1793-1 standard. The conducted acoustic tests results of which are presented by the authors in this paper show that preliminary acoustic tests of materials that make it possible to determine the values of their physical absorption coefficient using the standing wave method can be used to determine the single-number sound absorption evaluation index $D L_{\alpha}$ and the absorption property class. Experimental verification of tests conducted in the reverberation chamber confirmed compliance of results obtained with the use of both methods: the specified absorption property class of the studied materials, determined either on the basis of conversion of the physical absorption coefficient in $1 / 3$-octave bands into the reverberant coefficient or on the basis of measurement of the reverberant absorption coefficient, proved to be the same. It must only be clearly emphasized that it is very important to make both types of material samples in order to obtain compliance of these results: disks with diameter of $10 \mathrm{~cm}$ and $3 \mathrm{~cm}$ as well as samples with surface area of $10 \mathrm{~m}^{2}$ and the same technical parameters $[10,13]$. A significant factor is the method of filling the panels (expanded clay aggregate granulate) or, in the case of bulk samples (chip concrete), the method of surface formation of these panels. The proposed method of determining the absorption classes will definitely reduce the costs of designing acoustic barriers. This is very significant, particularly in the case of application of new materials in an acoustic barrier, in regard to which there is a margin of uncertainty of whether their sound-absorbing properties will fulfill the designer's expectations.

The authors of the present paper are not aware of any scientific research work that would present a comparison of the results of the reverberant absorption coefficient determined on the basis of the values of the physical absorption coefficient with the results obtained from tests in the reverberation chamber. The presented test results were inspired by demand reported by designers and manufacturers of acoustic barriers who, in the current economic situation, are searching for new and more effective methods of noise protection that could be used in design work with new materials, not used earlier, acous- tic properties of which are the same or better than those of the currently used conventional sound-absorbing materials being cheaper at the same time. In order to meet this challenge, designers and manufacturers are forced to bear high costs related to acoustic tests conducted in the reverberation chamber which is not always justified by the obtained results. These costs are not related to the tests themselves but above all, to fabrication of sufficiently large prototype barrier samples. The results of conducted experimental acoustic tests and conversion presented in this paper show the possibility to reduce the costs of acoustic barrier design.

During the conducted comparative acoustic tests results of which have been presented in this paper, the authors analyzed factors that have an influence on the compliance of obtained results. The analysis and conclusions on this subject will be presented in a separate report.

\section{Acknowledgments}

This work has been performed within the framework of statutory studies of the Department of Mechanics and Vibroacoustics of AGH during the years 2010 2013, Research task no. 5: "New material solutions of layered barriers in the design of vibroacoustic protection for machines and devices".

\section{References}

[1] PN-EN 1793-1:2001 - Anti-noise devices on roadways and motorways. Testing method for determination of acoustic properties. Part 1: Specific sound absorption curve.

[2] PN-EN 1793-3:2001 — Anti-noise devices on roadways and motorways. Testing method for determination of acoustic properties. Part 3: Normalized roadway noise spectrum.

[3] J. Sikora, Technical Magazine (Mechanics) 11-M, 89 (2007).

[4] J. Turkiewicz, J. Sikora, Insulation-constructionindustry-ecology 3,134, 66 (2009).

[5] P.M. Morse, Vibration and Sound, New York, McGraw-Hill, 1948.

[6] PN-EN ISO 354:2005 - Acoustics. Sound absorption measurement in a reverberation chamber.

[7] S. Fatima, A.R. Mohanty, Appl. Acoust. 72, 108 (2011).

[8] H. Kuttruff, Room Acoustics, Fifth edition, SponPress 2009.

[9] Handbook of Noise and Vibration Control, Ed. M.J. Crocker, John Wiley \& Sons, 2007.

[10] A. Chrobak, G. Wszołek, in: Proc. XX Conf. Acoust. Biomed. Eng. 2013, Kraków - Zakopane 2013, PTA, Kraków 2013, p. 157, (in Polish).

[11] K.U. Ingard, Notes on sound absorption technology, Noise Control Foundation, Poughkeepsie, New York 1994.

[12] J. Turkiewicz, Technical Magazine (Mechanics) 11M, 105 (2007).

[13] W.Bartnik, G. Wszołek, in: Proc. XX Conf. Acoust. Biomed. Eng. 2013, Kraków - Zakopane 2013, PTA, Kraków 2013, p. 152, (in Polish). 